\title{
BASES FOR CYCLOTOMIC UNITS OVER FUNCTION FIELDS
}

\author{
S. BAE and H. JUNG
}

(Received 15 February 1999; revised 11 April 2000)

\author{
Communicated by W. W. L. Chen
}

\begin{abstract}
We find a basis for the universal punctured even distribution and then a basis for the cyclotomic units over function fields.
\end{abstract}

2000 Mathematics subject classification: primary 11R58, $11 \mathrm{R} 60$.

\section{Introduction}

In the classical case, the structure of the group of cyclotomic units can be obtained from the universal punctured even distribution [6]. Gold and Kim [2] have found an explicit basis (a minimal set of generators) of the universal punctured even distribution and then, by eliminating some generators of it, a basis of the group of cyclotomic units. They used this basis to show that $U_{n}^{G}=U_{m}$ for all $m \mid n$ where $G=\operatorname{Gal}\left(\mathbb{Q}\left(\zeta_{n}\right) / \mathbb{Q}\left(\zeta_{m}\right)\right.$ ), and $U_{n}$ (respectively $U_{m}$ ) is the group of cyclotomic units in $\mathbb{Q}\left(\zeta_{n}\right)$ (respectively $\mathbb{Q}\left(\zeta_{m}\right)$ ).

Galovich and Rosen introduced the cyclotomic units in the cyclotomic function fields [3,4] and the distribution theory over function fields [5]. Bae described the group structure of universal even (odd) punctured distribution [1]. In this paper, we find a basis of the universal even punctured distribution in function field and from this basis find a basis of the group of cyclotomic units in the cyclotomic function fields, following the ideas of Gold and Kim [2].

Throughout this paper we fix the following notation. Since the case $q=2$ is not so interesting, we assume that $q>2$. 
Notation

$$
\begin{aligned}
\mathbb{F}_{q} & =\text { the finite field with } q \text { elements, } \\
R_{T} & =\mathbb{F}_{q}[T], \\
k & =\mathbb{F}_{q}(T), \\
R_{T}(M) & =(1 / M) R_{T} / R_{T}, \text { for a monic polynomial } M \text { of } R_{T}, \\
\lambda_{M} & =\text { a primitive } M \text {-th root of the Carlitz module } \rho, \\
\lambda_{M}^{A} & =\rho_{A}\left(\lambda_{M}\right), \\
\Phi(M) & =\text { the Euler totient function, } \\
\pi(M) & =\text { the number of monic irreducible divisors of } M, \\
k_{M} & =k\left(\lambda_{M}\right)=\text { the } M \text {-th cyclotomic function field over } k, \\
O_{M} & =\text { the integral closure of } R_{T} \text { in } k_{M}, \\
E_{M} & =\text { the group of units of } O_{M} .
\end{aligned}
$$

\section{Preliminaries}

Let $V_{M}$ be the subgroup of $k_{M}^{\times}$generated by

$$
\left\{\lambda_{M}^{A}: A \in R_{T} / M R_{T}, A \not \equiv 0 \bmod M\right\} \cup \mathbb{F}_{q}^{\times},
$$

and $U_{M}=E_{M} \cap V_{M}$ what is called the group of cyclotomic units of $k_{M}^{\times}$. It is well known [4] that $U_{M}$ is of finite index in $E_{M}$, in particular, they have the same rank $\Phi(M) /(q-1)-1$. There are relations among the elements of $V_{M}$

$$
\begin{aligned}
\lambda_{M}^{c A} & =c \lambda_{M}^{A}, \\
\lambda_{M}^{A N} & =\prod_{R} \lambda_{M}^{A+B R} \quad \text { if } B N=M,
\end{aligned}
$$

where $c \in \mathbb{F}_{q}^{\times}$and $R$ runs over all the polynomials whose degrees are less than $\operatorname{deg}(N)$. We begin by finding a basis of the universal punctured even distribution $\left(\mathrm{A}_{M}^{\circ}\right)^{+}$, which is an abelian group with generators

$$
\left\{g\left(\frac{A}{M}\right): \frac{A}{M} \in R_{T}(M), \frac{A}{M} \neq 0\right\}
$$

and relations

$$
\begin{aligned}
g\left(\frac{c A}{M}\right) & =g\left(\frac{A}{M}\right), \\
g\left(\frac{A}{N}\right) & =\sum_{R} g\left(\frac{A+R N}{M}\right) \quad \text { if } N \mid M \text { and } \frac{A}{N} \neq 0 .
\end{aligned}
$$


Define

$$
\varphi:\left(A_{M}^{\circ}\right)^{+} \longrightarrow V_{M} / \mathbb{F}_{q}^{x}
$$

by $\varphi(g(A / M))=\lambda_{M}^{A} \bmod \mathbb{F}_{q}^{\times}$. From the relations (2)-(4) and (5), we can easily see that $\varphi$ is a well-defined homomorphism.

In [1], Bae showed that

$$
\left(\mathrm{A}_{M}^{\circ}\right)^{+} \simeq \mathbb{Z}^{\Phi(M) /(q-1)+\pi(M)-1} \bigoplus(\mathbb{Z} /(q-1))^{2^{n(M)-1}-\pi(M)} .
$$

Therefore, we have the following theorem.

THEOREM 1.1. There is a split exact sequence

$$
0 \longrightarrow(\mathbb{Z} /(q-1))^{2^{r-1}-r} \longrightarrow\left(A_{M}^{\circ}\right)^{+} \stackrel{\varphi}{\longrightarrow} V_{M} / \mathbb{F}_{q}^{\times} \longrightarrow 0,
$$

where $\varphi$ is defined as above and $r=\pi(M)$.

\section{Basis of $\left(A_{M}^{\circ}\right)^{+}$}

Let $M$ be a monic polynomial and $Q_{1}^{e_{1}} Q_{2}^{e_{2}} \cdots Q_{r}^{e_{r}}$ be its factorization, with $Q_{i}$ monic irreducible. Let

$$
S=\left\{A \in R_{T}: \operatorname{deg} A<\operatorname{deg} M, A \text { is relatively prime to } M\right\},
$$

and define three subsets $S_{i}, \tilde{S}_{i}$ and $S_{i}^{*}$ of $S$ for each $i$ as follows:

$$
\begin{aligned}
& S_{i}=\left\{A \in S: A \equiv 1 \bmod M / Q_{i}^{e_{i}}\right\} \\
& \tilde{S}_{i}=\left\{A \in S_{i}: A \equiv \alpha \bmod Q_{i}^{e_{i}}, \text { for some } \alpha \in \mathbb{F}_{q}^{\times}\right\} \\
& S_{i}^{*}=\left\{A \in S_{i}: A \equiv \text { a monic polynomial of degree }<\operatorname{deg} Q_{i}^{e_{i}} \bmod Q_{i}^{e_{i}}\right\}
\end{aligned}
$$

For two elements $A$ and $B$ in $S_{i}$, we write $A B$ to denote the element of $S_{i}$ which is congruent to $A B \bmod M$. We also write $A^{-1}$ to denote an element $B$ of $S_{i}$ such that $A B \equiv 1 \bmod M$. Then every element of $S$ can be uniquely expressed as a product of $A_{1}, \ldots, A_{r}$, where $A_{i} \in S_{i}$. Let $\alpha$ be a generator of $\mathbb{F}_{q}^{\times}$. We choose $\tilde{A}_{i} \in \tilde{S}_{i}$ such that $\alpha \equiv \tilde{A}_{i} \bmod Q_{i}^{e_{i}}$.

LEMMA 2.1. Suppose that $(B, M)=1$. Then

$$
\sum_{A \in S_{i}} g\left(\frac{B A}{M}\right)=g\left(\frac{B}{M / Q_{i}^{e_{i}}}\right)-g\left(\frac{C}{M / Q_{i}^{e_{i}}}\right) \in\left(A_{M / Q_{i}^{\varepsilon_{i}}}^{0}\right)^{+}
$$

for some $C$. 
PROOF. The same procedure as in the classical case [2] gives the result.

Let

$$
I_{M}=\left\{\left(A_{1}, \ldots, A_{r}\right): A_{i} \in S_{i} \text { for } i \leq r-1 \text {, and } A_{r} \in S_{r}^{*}\right\}
$$

and

$I_{M}^{\prime}=\left\{\left(A_{1}, \ldots, A_{r}\right) \in I_{M}\right.$ satisfying one of the following conditions:

- $A_{r} \in S_{r}^{*} \backslash\{1\}$ and $A_{i} \in S_{i} \backslash\{1\}$ for $i \leq r-1$;

- when $r-l$ is odd, $A_{r}=\cdots=A_{l+1}=1, A_{l} \in\left(S_{l} \backslash\{1\}\right) \backslash \tilde{S}_{l}^{*}$ and $A_{i} \in S_{i} \backslash\{1\}$ for $i \leq l-1$;

- when $r-l$ is even, $A_{r}=\cdots=A_{l+1}=1, A_{l} \in S_{l}^{*} \backslash\{1\}$ and $A_{i} \in S_{i} \backslash\{1\}$, for $i \leq l-1$;

- $\left.A_{r}=\cdots=A_{1}=1.\right\}$

Here $\tilde{S}_{l}^{*}=\left\{A \in S_{l}: A \equiv \tilde{A}_{l} B \bmod M\right.$ for some $\left.B \in S_{l}^{*}\right\}$.

REMARK. The difference in the definition of $I_{M}^{\prime}$ from that in [2] arises from the fact that there are $(q-1)$ roots of unity. In fact, if $q=3$, then $\left(S_{l} \backslash\{1\}\right) \backslash \tilde{S}_{l}^{*}$ is $S_{l}^{*} \backslash\{1\}$.

LEMMA 2.2. The cardinality $\left|I_{M}^{\prime}\right|$ of the set $I_{M}^{\prime}$ is as follows:

(i) If $r$ is even, $\left|I_{M}^{\prime}\right|=1 /(q-1) \prod_{i=1}^{r}\left(\Phi\left(Q_{i}^{e_{i}}\right)-1\right)+(q-2) /(q-1)$.

(ii) If $r$ is odd, $\left|I_{M}^{\prime}\right|=1 /(q-1) \prod_{i=1}^{r}\left(\Phi\left(Q_{i}^{e_{i}}\right)-1\right)+1 /(q-1)$.

In either case, we have $\sum_{D}\left|I_{D}^{\prime}\right|=\Phi(M) /(q-1)+2^{r-1}-1$, where $D$ runs over all monic divisors of $M$ such that $(D, M / D)=1$ and $D \neq 1$.

PROOF. We prove the case that $r$ is even. The case where $r$ is odd is very similar and we leave it to the reader. Suppose that $r$ is even and let $X_{i}=\Phi\left(Q_{i}^{e_{i}}\right)-1$ for $1 \leq i \leq r$. From the definition of $I_{M}^{\prime}$, we have

$$
\begin{aligned}
\left|I_{M}^{\prime}\right|= & \left(\frac{X_{r}+1}{q-1}-1\right) \prod_{i=1}^{r-1} X_{i}+\left(\frac{q-2}{q-1}\left(X_{r-1}+1\right)-1\right) \prod_{i=1}^{r-2} X_{i}+\cdots \\
& +\left(\frac{X_{2}+1}{q-1}-1\right) X_{1}+\left(\frac{q-2}{q-1}\left(X_{1}+1\right)-1\right)+1
\end{aligned}
$$

By expanding above one, we get (i). Note that there are $\left(\begin{array}{l}r \\ l\end{array}\right)$ distinct $D^{\prime}$ s such that $\pi(D)=l$ for $1 \leq l \leq r$ and that

$$
\Phi(M)=\prod_{i=1}^{r}\left(X_{i}+1\right)=\sum_{i=1}^{r}\left(\sum_{I \leq i_{1}<\cdots<i_{i} \leq r} X_{i_{1}} \cdots X_{i_{i}}\right) .
$$


The results in (i), (ii) and elementary calculation show that

$$
\sum_{D}\left|I_{D}^{\prime}\right|=\frac{\Phi(M)}{q-1}+2^{r-1}-1 .
$$

Let $T_{M}$ be the subgroup of $\left(\mathrm{A}_{M}^{\circ}\right)^{+}$generated by

and let

$$
\left\{g\left(\frac{A_{1} \cdots A_{r}}{M}\right):\left(A_{1}, \ldots, A_{r}\right) \in I_{M}^{\prime}\right\}
$$

$$
T_{M}^{\prime}=\prod_{D} T_{D}
$$

where $D$ runs over all monic divisors of $M$ such that $(D, M / D)=1, D \neq 1, M$, and $T_{D}$ is defined similarly to $T_{M}$.

THEOREM 2.3.

$$
\left(\mathrm{A}_{M}^{\circ}\right)^{+}=T_{M} \times T_{M}^{\prime}=\prod_{D} T_{D}
$$

where $D$ runs over all monic divisors of $M$ such that $(D, M / D)=1$ and $D \neq 1$.

REMARK. As we have shown in Lemma 2.2, $\sum_{D}\left|I_{D}^{\prime}\right|=\Phi(M) /(q-1)+2^{\pi(M)-1}-1$, which is the minimum number of generators of $\left(A_{M}^{\circ}\right)^{+}$. Theorem 2.3 provides a basis (minimal set of generators) of $\left(A_{M}^{\circ}\right)^{+}$.

PROOF OF THEOREM 2.3. We use induction on $r$. The case $r=1$ is trivial. Assume the theorem holds for $M$ with $\pi(M) \leq r-1$. We prove $g\left(A_{1} \cdots A_{r} / M\right) \in T_{M} \times T_{M}^{\prime}$ for $\left(A_{1}, \ldots, A_{r}\right) \in I_{M} \backslash I_{M}^{\prime}$ case by case.

(i) If $A_{l} \in\left(S_{l} \backslash\{1\}\right) \backslash \tilde{S}_{l}^{*}$ for $r-l$ odd, or $A_{l} \in S_{l}^{*} \backslash\{1\}$ for $r-l$ even, then

$$
g\left(\frac{A_{1} \cdots A_{l}}{M}\right) \in T_{M} \times T_{M}^{\prime}
$$

Proof. When none of $A_{i}^{\prime} s$ is $1,\left(A_{1}, \ldots, A_{l}\right) \in I_{M}^{\prime}$, then $g\left(A_{1} \cdots A_{l} / M\right) \in T_{M}$. Suppose exactly one of $A_{i}$ is 1 , say $A_{t}=1$. Then by the relation (6) in Lemma 2.1,

$$
\sum_{B \in S_{t}} g\left(\frac{A_{1} \cdots A_{t-1} B A_{t+1} \cdots A_{l}}{M}\right) \in T_{M}^{\prime} .
$$

Since $g\left(A_{1} \cdots A_{t-1} B A_{t+1} \cdots A_{l} / M\right) \in T_{M}$ for all $B \neq 1$ by the previous case, $g\left(A_{1} \cdots A_{t-1} 1 A_{t+1} \cdots A_{l} / M\right) \in T_{M} \times T_{M}^{\prime}$. Now proceed exactly the same way as in the classical case (case (i) or (ii) of the proof of [2, Theorem 1]). 
(ii) If $A_{l} \notin\left\{1, \tilde{A}_{l}\right\}$ for $r-l$ odd, or $A_{l} \notin \tilde{S}_{l}$ for $r-l$ even, then

$$
g\left(\frac{A_{1} \cdots A_{l}}{M}\right) \in T_{M} \times T_{M}^{\prime}
$$

PROOF. When $l=r$, it suffices to show that for $A_{r}^{*} \in S_{r}^{*}, A_{r}^{*} \neq 1$

$$
g\left(\frac{A_{1} \cdots A_{r-1} A_{r}^{*} \tilde{A}_{r}^{t}}{M}\right) \in T_{M} \times T_{M}^{\prime}
$$

But by case (i), we have

$$
g\left(\frac{A_{1} \cdots A_{r-1} A_{r}^{*} \tilde{A}_{r}^{t}}{M}\right)=g\left(\frac{A_{1} \tilde{A}_{1}^{-t} \cdots A_{r-1} \tilde{A}_{r-1}^{-t} A_{r}^{*}}{M}\right) \in T_{M} \times T_{M}^{\prime} .
$$

When $l=r-1$, by the definition of $I_{M}^{\prime}$, it suffices to show that for $A_{r-1}^{*} \in S_{r-1}^{*}$, $A_{r-1}^{*} \neq 1$

$$
g\left(\frac{A_{1} \cdots A_{r-1}^{*} \tilde{A}_{r-1}}{M}\right) \in T_{M} \times T_{M}^{\prime} .
$$

By the relation (6) in Lemma 2.1, we have

$$
\begin{aligned}
& g\left(\frac{A_{1} \cdots A_{r-1}^{*} \tilde{A}_{r-1}}{M}\right)+\sum_{t=1}^{q-2} g\left(\frac{A_{1} \cdots A_{r-1}^{*} \tilde{A}_{r-1} \tilde{A}_{r}^{t}}{M}\right)+\sum_{\substack{A_{*}^{*} \in S_{r}^{*} \\
A_{z}^{*} \neq \neq 1}} g\left(\frac{A_{1} \cdots A_{r-1}^{*} \tilde{A}_{r-1} A_{r}^{*}}{M}\right) \\
& +\sum_{\substack{A_{r}^{*} \in S_{r}^{*} \\
A_{r}^{*} \neq 1}} \sum_{t=1}^{q-2} g\left(\frac{A_{1} \cdots A_{r-1}^{*} \tilde{A}_{r-1} A_{r}^{*} \tilde{A}_{r}^{t}}{M}\right) \in T_{M}^{\prime}
\end{aligned}
$$

Note that

$$
g\left(\frac{A_{1} \cdots A_{r-1}^{*} \tilde{A}_{r-1} \tilde{A}_{r}^{t}}{M}\right)=g\left(\frac{A_{1} \tilde{A}_{1}^{-1} \cdots A_{r-1}^{*} \tilde{A}_{r-1}^{1-t}}{M}\right) \in T_{M} \times T_{M}^{\prime}
$$

because $\tilde{A}_{r-1}^{1-t} \neq 1, \tilde{A}_{r-1}$ for $t \neq 1$ and $g\left(A_{1} \tilde{A}_{1}^{-1} \cdots A_{r-1}^{*} / M\right) \in T_{M} \times T_{M}^{\prime}$ for $t=1$. All terms except $g\left(A_{1} \cdots A_{r-1}^{*} \tilde{A}_{r-1} / M\right)$ are contained in $T_{M} \times T_{M}^{\prime}$, by case (i) and the previous note, so $g\left(A_{1} \cdots A_{r-1}^{*} \tilde{A}_{r-1} / M\right)$ is contained in $T_{M} \times T_{M}^{\prime}$.

Now we assume that the assertion is true for $l+1, l+2, \ldots, r$ and we prove the case $r-l$ is odd. The case $r-l$ is even is very similar and we leave it to the reader. It is enough to show that for $A_{l}^{*} \in S_{l}^{*}$ and $A_{l}^{*} \neq 1$

$$
g\left(\frac{A_{1} \cdots A_{l}^{*} \tilde{A}_{l}}{M}\right) \in T_{M} \times T_{M}^{\prime}
$$


By the relation (6) in Lemma 2.1, we have

$$
\begin{aligned}
& g\left(\frac{A_{1} \cdots A_{l}^{*} \tilde{A}_{l}}{M}\right)+\sum_{\alpha_{l+1}=1}^{q-2} g\left(\frac{A_{1} \cdots A_{l}^{*} \tilde{A}_{l} \tilde{A}_{l+1}^{\alpha_{l+1}}}{M}\right)+\sum_{\substack{A_{i+1}^{*} \in S_{i+1}^{*} \\
A_{i+1}^{*} \neq 1}} g\left(\frac{A_{1} \cdots A_{l}^{*} \tilde{A}_{l} A_{l+1}^{*}}{M}\right) \\
& +\sum_{\substack{A_{i+1} \in S_{i+1}^{*} \\
A_{i+1} \neq 1}} \sum_{\alpha_{l+1}=1}^{q-2} g\left(\frac{A_{1} \cdots A_{l}^{*} \tilde{A}_{l} A_{l+1}^{*} \tilde{A}_{l+1}^{\alpha_{i+1}}}{M}\right) \in T_{M}^{\prime}
\end{aligned}
$$

Note that the last two sums are contained in $T_{M} \times T_{M}^{\prime}$ by case (i) and the inductive hypothesis. Hence (7) is equivalent to

$$
\sum_{\alpha_{l+1}=1}^{q-2} g\left(\frac{A_{1} \cdots A_{l}^{*} \tilde{A}_{l} \tilde{A}_{l+1}^{\alpha_{l+1}}}{M}\right) \in T_{M} \times T_{M}^{\prime} .
$$

Apply the relation (6) in Lemma 2.1 again, we have

$$
\begin{aligned}
g\left(\frac{A_{1} \cdots A_{l}^{*} \tilde{A}_{l} \tilde{A}_{l+1}^{\alpha_{l+1}}}{M}\right) & +\sum_{\alpha_{l+2}=1}^{q-2} g\left(\frac{A_{1} \cdots A_{l}^{*} \tilde{A}_{l} \tilde{A}_{l+1}^{\alpha_{l+1}} \tilde{A}_{l+2}^{\alpha_{l+2}}}{M}\right) \\
& +\sum_{\substack{A_{i+2} \in S_{l+2}^{*} \\
A_{i+2} \neq 1}} g\left(\frac{A_{1} \cdots A_{l}^{*} \tilde{A}_{l} \tilde{A}_{l+1}^{\alpha_{l+1}} A_{l+2}^{*}}{M}\right) \\
& +\sum_{\substack{A_{i+2}^{*} \in S_{l+2}^{*} \\
A_{i+2}^{*} \neq 1}} \sum_{\alpha_{l+2}=1}^{q-2} g\left(\frac{A_{1} \cdots A_{l}^{*} \tilde{A}_{l} \tilde{A}_{l+1}^{\alpha_{l+1}} A_{l+2}^{*} \tilde{A}_{l+2}^{\alpha_{l+2}}}{M}\right) \in T_{M}^{\prime} .
\end{aligned}
$$

The last two sums are contained in $T_{M} \times T_{M}^{\prime}$ by case (i) and the inductive hypothesis and

$$
g\left(\frac{A_{1} \cdots A_{l}^{*} \tilde{A}_{l} \tilde{A}_{l+1}^{\alpha_{l+1}} \tilde{A}_{l+2}^{\alpha_{l+2}}}{M}\right) \in T_{M} \times T_{M}^{\prime}
$$

for all $\alpha_{l+2} \neq 1$ by the inductive hypothesis (note that $r-(l+2)$ is odd). Hence

$$
\sum_{\alpha_{l+1}=1}^{q-2} g\left(\frac{A_{1} \cdots A_{l}^{*} \tilde{A}_{l} \tilde{A}_{l+1}^{\alpha_{l+1}}}{M}\right) \in T_{M} \times T_{M}^{\prime}
$$

is equivalent to

$$
\sum_{\alpha_{l+1}=1}^{q-2} g\left(\frac{A_{1} \cdots A_{l}^{*} \tilde{A}_{l} \tilde{A}_{l+1}^{\alpha_{l+1}} \tilde{A}_{l+2}}{M}\right) \in T_{M} \times T_{M}^{\prime}
$$


Repeating the above procedure, we see that (7) is equivalent to

$$
\sum_{\alpha_{r}=1}^{q-2} \cdots \sum_{\alpha_{i+1}=1}^{q-2} g\left(\frac{A_{1} \cdots A_{l}^{*} \tilde{A}_{l} \tilde{A}_{l+1}^{\alpha_{l+1}} \cdots \tilde{A}_{r-1} \tilde{A}_{r}^{\alpha_{r}}}{M}\right) \in T_{M} \times T_{M}^{\prime} .
$$

We claim that, for $1 \leq \alpha_{i} \leq q-2$,

$$
g\left(\frac{A_{1} \cdots A_{l}^{*} \tilde{A}_{l} \tilde{A}_{l+1}^{\alpha_{l+1}} \tilde{A}_{l+1} \cdots \tilde{A}_{r-2}^{\alpha_{r-2}} \tilde{A}_{r-1} \tilde{A}_{r}^{\alpha_{r}}}{M}\right) \in T_{M} \times T_{M}^{\prime}
$$

if $\alpha_{t} \neq 1$ for some $t=l+1, \ldots, r-2, r$.

PROOF OF CLAIM. When $\alpha_{r} \neq 1$, the left hand side of (9) is equal to

$$
g\left(\frac{A_{1} \tilde{A}_{1}^{-\alpha_{r}} \cdots A_{l}^{*} \tilde{A}_{l}^{1-\alpha_{r}} \cdots \tilde{A}_{r-1}^{1-\alpha_{r}}}{M}\right)
$$

which is contained in $T_{M} \times T_{M}^{\prime}$, because $\tilde{A}_{r-1}^{1-\alpha_{r}} \neq 1, \tilde{A}_{r-1}$. Now we suppose that the assertion is true for $t+2, \ldots, r-2, r$ and we show that for $\alpha_{t} \neq 1$,

$$
g\left(\frac{A_{1} \cdots A_{l}^{*} \tilde{A}_{l} \tilde{A}_{l+1}^{\alpha_{l+1}} \cdots \tilde{A}_{t-1} \tilde{A}_{t}^{\alpha_{t}} \tilde{A}_{t+1} \cdots \tilde{A}_{r-2} \tilde{A}_{r-1} \tilde{A}_{r}}{M}\right) \in T_{M} \times T_{M}^{\prime}
$$

which is equal to

$$
g\left(\frac{A_{1} \tilde{A}_{1}^{-1} \cdots A_{l}^{*} \tilde{A}_{l+1}^{\alpha_{l+1}-1} \cdots \tilde{A}_{t-2}^{\alpha_{t-2}-1} \tilde{A}_{t}^{\alpha_{t}-1}}{M}\right) .
$$

By case (i), the inductive hypothesis of proof of case (ii) and the inductive hypothesis of the claim (using relation (6) in Lemma 2.1 repeatedly), (10) is equivalent to

$$
g\left(\frac{A_{1} \tilde{A}_{1}^{-1} \cdots A_{l}^{*} \tilde{A}_{l+1}^{\alpha_{t+1}-1} \cdots \tilde{A}_{t-2}^{\alpha_{t-2}-1} \tilde{A}_{t}^{\alpha_{t}-1} \tilde{A}_{t+1} \cdots \tilde{A}_{r-1} \tilde{A}_{r}}{M}\right) \in T_{M} \times T_{M}^{\prime} .
$$

Note that (11) is equal to

$$
g\left(\frac{A_{1} \tilde{A}_{1}^{-2} \cdots A_{l}^{*} \tilde{A}_{l+1}^{\alpha_{l+1}-2} \cdots \tilde{A}_{t-2}^{\alpha_{t-2}-2} \tilde{A}_{t-1}^{-1} \tilde{A}_{t}^{\alpha_{t}-2}}{M}\right) .
$$

Repeating this procedure, we see that $(10)$ is equivalent to

$$
g\left(\frac{A_{1} \tilde{A}_{1}^{-\alpha_{i}} \cdots A_{l}^{*} \tilde{A}_{l}^{1-\alpha_{l}} \cdots \tilde{A}_{t-1}^{1-\alpha_{i}}}{M}\right) \in T_{M} \times T_{M}^{\prime},
$$

which is true because $\tilde{A}_{t-1}^{1-\alpha_{1}} \neq 1, \tilde{A}_{t-1}$ (note that $r-t$ is even and $t>l$ ). This proves our claim. 
Now we return to the proof of case (ii). By (9), (8) is equivalent to

$$
g\left(\frac{A_{1} \cdots A_{l}^{*} \tilde{A}_{l} \tilde{A}_{l+1} \cdots \tilde{A}_{r-1} \tilde{A}_{r}}{M}\right) \in T_{M} \times T_{M}^{\prime} .
$$

But this term is equal to $g\left(A_{1} \tilde{A}^{-1} \cdots A_{l}^{*} / M\right)$ which is contained in $T_{M} \times T_{M}^{\prime}$.

REMARK. The claim in the proof of (ii) is true under the inductive hypothesis of (ii). However, we have proven (ii) anyway, the claim is true in more general setting, that is,

$$
g\left(\frac{A_{1} \cdots A_{l} \tilde{A}_{l+1}^{\alpha_{l+1}} \tilde{A}_{l+2} \cdots \tilde{A}_{r-1} \tilde{A}_{r}^{\alpha_{r}}}{M}\right) \in T_{M} \times T_{M}^{\prime},
$$

if $r-l$ is odd and $1 \leq \alpha_{t} \leq q-2, \alpha_{t} \neq 1$ for some $t$ and

$$
g\left(\frac{A_{1} \cdots A_{l} \tilde{A}_{l+1} \tilde{A}_{l+2}^{\alpha_{l+2}} \cdots \tilde{A}_{r-1} \tilde{A}_{r}^{\alpha_{r}}}{M}\right) \in T_{M} \times T_{M}^{\prime},
$$

if $r-l$ is even and $1 \leq \alpha_{t} \leq q-2, \alpha_{t} \neq 1$ for some $t$.

To prove the remaining cases, we need the following lemma.

LEMMA 2.4. The following two statements are equivalent:

(i) $g\left(A_{1} \cdots A_{l-1}\left(A_{l} \tilde{A}_{l}\right) / M\right) \in T_{M} \times T_{M}^{\prime}$;

(ii) $g\left(\left(A_{1} \tilde{A}_{1}^{-1}\right) \cdots\left(A_{l-1} \tilde{A}_{l-1}^{-1}\right) A_{l} / M\right) \in T_{M} \times T_{M}^{\prime}$.

PROOF. We prove this lemma for the case $r-l$ odd. By the relation (6) in Lemma 2.1,

$$
\sum_{B \in S_{l+1}} g\left(\frac{A_{1} \cdots A_{l} \tilde{A}_{l} B}{M}\right) \in T_{M}^{\prime} .
$$

Since $g\left(A_{1} \cdots A_{l} \tilde{A}_{l} B / M\right) \in T_{M}^{\prime}$ for all $B \notin \tilde{S}_{l+1}$, by case (ii), we have

$$
g\left(\frac{A_{1} \cdots A_{l} \tilde{A}_{l}}{M}\right)+\sum_{\alpha_{l+1}=1}^{q-2} g\left(\frac{A_{1} \cdots A_{l} \tilde{A}_{l} \tilde{A}_{l+1}^{\alpha_{l+1}}}{M}\right) \in T_{M} \times T_{M}^{\prime} .
$$

Hence

$$
g\left(\frac{A_{1} \cdots A_{l} \tilde{A}_{l}}{M}\right) \in T_{M} \times T_{M}^{\prime}
$$

is equivalent to

$$
\sum_{\alpha_{l+1}=1}^{q-2} g\left(\frac{A_{1} \cdots A_{l} \tilde{A}_{l} \tilde{A}_{l+1}^{\alpha_{l+1}}}{M}\right) \in T_{M} \times T_{M}^{\prime}
$$


We note that $r-(l+1)$ is even and use the relation (6) in Lemma 2.1 again, then we see that

$$
\sum_{\alpha_{l+1}=1}^{q-2} g\left(\frac{A_{1} \cdots A_{l} \tilde{A}_{l} \tilde{A}_{l+1}^{\alpha_{l+1}}}{M}\right) \in T_{M} \times T_{M}^{\prime}
$$

is equivalent to

$$
\sum_{\alpha_{l+1}=1}^{q-2} g\left(\frac{A_{1} \cdots A_{l} \tilde{A}_{l} \tilde{A}_{l+1}^{\alpha_{l+1}} \tilde{A}_{l+2}}{M}\right) \in T_{M} \times T_{M}^{\prime} .
$$

Therefore, (12) is equivalent to

$$
\sum_{\alpha_{l+1}=1}^{q-2} g\left(\frac{A_{1} \cdots A_{l} \tilde{A}_{l} \tilde{A}_{l+1}^{\alpha_{l+1}} \tilde{A}_{l+2}}{M}\right) \in T_{M} \times T_{M}^{\prime} .
$$

Repeating the above procedure, we can see that

$$
g\left(\frac{A_{1} \cdots A_{l} \tilde{A}_{l}}{M}\right) \in T_{M} \times T_{M}^{\prime}
$$

is equivalent to

$$
\sum_{\alpha_{r}=1}^{q-2} \cdots \sum_{\alpha_{l+1}=1}^{q-2} g\left(\frac{A_{1} \cdots A_{l} \tilde{A}_{l} \tilde{A}_{l+1}^{\alpha_{l+1}} \cdots \tilde{A}_{r-1} \tilde{A}_{r}^{\alpha_{r}}}{M}\right) \in T_{M} \times T_{M}^{\prime} .
$$

By the remark above,

$$
g\left(\frac{A_{1} \cdots A_{l} \tilde{A}_{l} \tilde{A}_{l+1}^{\alpha_{l+1}} \cdots \tilde{A}_{r-1} \tilde{A}_{r}^{\alpha_{r}}}{M}\right) \in T_{M} \times T_{M}^{\prime}
$$

for all $\left(\alpha_{l+1}, \ldots, \alpha_{r}\right) \neq(1, \ldots, 1)$. Hence (13) is equivalent to

$$
g\left(\frac{A_{1} \cdots A_{l} \tilde{A}_{l} \tilde{A}_{l+1} \cdots \tilde{A}_{r-1} \tilde{A}_{r}}{M}\right) \in T_{M} \times T_{M}^{\prime}
$$

But $g\left(A_{1} \cdots A_{l} \tilde{A}_{l} \tilde{A}_{l+1} \cdots \tilde{A}_{r-1} \tilde{A}_{r} / M\right)=g\left(A_{1} \tilde{A}_{1}^{-1} \cdots A_{l} / M\right)$, so we get the result.

We return to the proof of Theorem 2.3.

(iii) If $\left(A_{1}, \ldots, A_{r-1}\right) \notin \tilde{S}_{1} \times \cdots \times \tilde{S}_{r-1}$, then

$$
g\left(\frac{A_{1} A_{2} \cdots A_{r-1}}{M}\right) \in T_{M} \times T_{M}^{\prime}
$$

PROOF. It suffices to show that

$$
g\left(\frac{A_{1} \cdots A_{l}^{*} \tilde{A}_{l}^{t} \tilde{A}_{l+1}^{t_{l+1}} \cdots \tilde{A}_{r-1}^{t_{r-1}}}{M}\right) \in T_{M} \times T_{M}^{\prime}
$$


for any $l$ with $A_{l}^{*} \neq 1$. By Lemma 2.4 , it is equivalent to

$$
g\left(\frac{A_{1} \tilde{A}_{1}^{-\ell} \cdots A_{l}^{*}}{M}\right) \in T_{M} \times T_{M}^{\prime},
$$

where $t=\sum_{i=l}^{r-1} t_{i}$. But it is true by case (i).

(iv) $g\left(\tilde{A}_{1}^{t_{1}} \cdots \tilde{A}_{r-1}^{t_{r-1}} / M\right) \in T_{M} \times T_{M}^{\prime}$.

Proof. By Lemma 2.4, it suffices to show that

$$
g\left(\frac{\tilde{A}_{1}^{\alpha}}{M}\right) \in T_{M} \times T_{M}^{\prime}, \quad \forall \alpha=0,1, \ldots, q-2
$$

If $q=3$, then $g\left(\tilde{A}_{1} / M\right)+g(1 / M) \in T_{M} \times T_{M}^{\prime}$, by (ii) and Lemma 2.4. Since $g(1 / M) \in T_{M} \times T_{M}^{\prime}, g\left(\tilde{A}_{1} / M\right) \in T_{M} \times T_{M}^{\prime}$. Now assume that $q>3$. If $r$ is even, then $r-1$ is odd. In this case

$$
g\left(\frac{1}{M}\right)+g\left(\frac{\tilde{A}_{1}}{M}\right)+\sum_{\alpha=2}^{q-2} g\left(\frac{\tilde{A}_{1}^{\alpha}}{M}\right) \in T_{M} \times T_{M}^{\prime} .
$$

But $g\left(\tilde{A}_{1}^{\alpha} / M\right) \in T_{M} \times T_{M}^{\prime}$ for $\alpha=2, \ldots, q-1$ by (ii), $g(1 / M) \in T_{M} \times T_{M}^{\prime}$, so $g\left(\tilde{A}_{1} / M\right) \in T_{M} \times T_{M}^{\prime}$. If $r$ is odd, then $r-1$ is even. By Lemma $2.4, g\left(\tilde{A}_{1}^{\alpha} / M\right) \epsilon$ $T_{M} \times T_{M}^{\prime}$ is equivalent to $g\left(\tilde{A}_{1}^{\alpha+2} \tilde{A}_{2}^{2} / M\right) \in T_{M} \times T_{M}^{\prime}$ which is true by (ii), since $r-2$ is odd.

\section{Basis of $U_{M}$}

Let $M=Q_{1}^{e_{1}} Q_{2}^{e_{2}} \cdots Q_{r}^{e_{r}}$ be as before. To find a basis of $U_{M}$ we eliminate certain generators of $T_{M}$. To be precise, let

$$
\begin{aligned}
I_{M}^{\prime \prime} & = \begin{cases}I_{M}^{\prime}-\{(1, \ldots, 1)\} & \text { if } r \text { is odd; } \\
I_{M}^{\prime} & \text { if } r \text { is even. }\end{cases} \\
\tilde{g}\left(\frac{A}{M}\right) & = \begin{cases}g(A / M) & \text { if } M \text { is composite; } \\
g\left(A / Q^{e}\right)-g\left(1 / Q^{e}\right) & \text { if } M=Q^{e} .\end{cases}
\end{aligned}
$$

Let $\tilde{T}_{M}$ be the group generated by the elements $\tilde{g}\left(A_{1} \cdots A_{r} / M\right)$ with $\left(A_{1}, \ldots, A_{r}\right) \in$ $I_{M}^{\prime \prime}$ and $\tilde{T}_{M}^{\prime}=\prod_{D} \tilde{T}_{D}$, where $D$ runs over all monic divisors of $M$ such that $(D, M / D)=1, D \neq 1, M$ and $\tilde{T}_{D}$ is defined similarly to $\tilde{T}_{M}$. Then we have

$$
\left(\mathrm{A}_{M}^{\circ}\right)^{+}=G_{1} \times G_{2} \times G_{3}
$$


where

$$
\begin{aligned}
& G_{1}=\tilde{T}_{M} \times \tilde{T}_{M}^{\prime}, \\
& G_{2}=\text { group generated by }\left\{g\left(1 / Q_{i}^{e_{i}}\right): 1 \leq i \leq r\right\} \\
& G_{3}=\text { group generated by }\left\{g\left(1 / Q_{i_{1}}^{e_{i_{1}}} \cdots Q_{i_{l}}^{e_{i_{l}}}\right): l \geq 3, \text { odd }\right\} .
\end{aligned}
$$

LEMMA 3.1.

$$
\sum_{D}\left|I_{D}^{\prime \prime}\right|=\frac{\Phi(M)}{q-1}-1
$$

where $D$ runs over all monic divisor of $M$ such that $(D, M / D)=1$ and $D \neq 1$.

PROOF. We only prove the case that $r$ is even. Since the case $r$ is odd is very similar, we leave it to the reader. When $r$ is even, we have

$$
\sum_{D}\left|I_{D}^{\prime \prime}\right|=\sum_{D}\left|I_{D}^{\prime}\right|-\sum_{\substack{1 \leq l \leq r-1 \\
l \text { odd }}}\left(\begin{array}{c}
r \\
r-l
\end{array}\right)
$$

and $\sum_{\substack{1 \leq l \leq r-1 \\ l \text { odd }}}\left(\begin{array}{c}r \\ r-l\end{array}\right)$ is $2^{r-1}$. Hence the result follows.

LEMMA 3.2. $(q-1) g(1 / M) \in G_{1}$, if $M$ is a composite.

PROOF. If $r$ is even there is nothing to prove. So we assume $r$ is odd. Let

$$
R_{0}=\sum_{A_{1}^{*} \in S_{1}^{*}} \sum_{A_{2}^{*} \in S_{2}^{*}} \cdots \sum_{A_{r}^{*} \in S_{r}^{*}} g\left(\frac{A_{1}^{*} A_{2}^{*} \cdots A_{r}^{*}}{M}\right)
$$

and for each $l, 1 \leq l \leq r$, let

$$
R_{l}=\sum_{\substack{A_{i}^{*} \in S_{1}^{*} \\ 1 \leq \alpha_{1} \leq q-2}} \cdots \sum_{\substack{A_{i} \in S_{l}^{*} \\ 1 \leq \alpha_{l} \leq q-2}} \sum_{A_{i+1}^{*} \in S_{l+1}^{*}} \cdots \sum_{A_{i}^{*} \in S_{r}^{*}} g\left(\frac{A_{1}^{*} \tilde{A}_{1}^{\alpha_{l}} \cdots A_{l}^{*} \tilde{A}_{l}^{\alpha_{l}} A_{l+1}^{*} \cdots A_{r}^{*}}{M}\right)
$$

Then by Lemma 2.1 , we have $R_{i}+R_{i+1} \in \tilde{T}_{M}^{\prime}$ for each $i=0,1, \ldots, r-1$ and so $R_{0}+R_{r}=\left(R_{0}+R_{1}\right)-\left(R_{1}+R_{2}\right)+\cdots+\left(R_{r-1}+R_{r}\right) \in \tilde{T}_{M}^{\prime}$. If $\left(A_{1}^{*}, \ldots, A_{r}^{*}\right) \neq$ $(1, \ldots, 1)$,

$$
g\left(\frac{A_{1}^{*} A_{2}^{*} \cdots A_{r}^{*}}{M}\right) \in T_{M} \times T_{M}^{\prime}
$$

and if $\left(A_{1}^{*}, \ldots, A_{r}^{*}\right) \neq(1, \ldots, 1)$ or $\alpha_{i} \neq \alpha_{j}$ for some $i \neq j$,

$$
g\left(\frac{A_{1}^{*} \tilde{A}_{1}^{\alpha_{1}} \cdots A_{r}^{*} \tilde{A}_{r}^{\alpha_{r}}}{M}\right) \in T_{M} \times T_{M}^{\prime}
$$


Thus $R_{0}+R_{r} \in \tilde{T}_{M}^{\prime}$ implies

$$
g\left(\frac{1}{M}\right)+\sum_{1 \leq \alpha \leq q-2} g\left(\frac{\tilde{A}_{1}^{\alpha} \cdots \tilde{A}_{r}^{\alpha}}{M}\right) \in T_{M} \times T_{M}^{\prime} .
$$

But $g\left(\tilde{A}_{1}^{\alpha} \cdots \tilde{A}_{r}^{\alpha} / M\right)=g(1 / M)$, so the result follows.

LEMMA 3.3. The given generators of $G_{1} \times G_{2}$ are linearly independent over $\mathbb{Z}$.

PROOF. Almost the same proof as in [2] gives the result.

LEMMA 3.4. Let $r \geq 3$ odd. Then there exist a unique $R \in\left(\mathrm{A}_{M}^{0}\right)^{+}$such that $R \neq 0$, $(q-1) R=0$ and $R$ is of the form

$$
R=g\left(\frac{1}{M}\right)+\sum_{\tilde{g}(A / M) \in G_{1}} \tilde{f}\left(\frac{A}{M}\right) \tilde{g}\left(\frac{A}{M}\right)
$$

with $\tilde{f}(A / M) \in \mathbb{Z}$.

PROOF. Uniqueness is immediate by Lemma 3.3. We prove existence by induction on $r$. Suppose that $r=3$. In this case Tor $\left(\left(A_{M}^{0}\right)^{+}\right) \simeq \mathbb{Z} /(q-1)$, so there is $R \neq 0$ such that $(q-1) R=0$. Since $\left(\mathrm{A}_{M}^{0}\right)^{+}=G_{1} \times G_{2} \times G_{3}$, we may write

$$
R=m g\left(\frac{1}{M}\right)+\sum_{\tilde{g}(A / M) \in G_{1}} \tilde{f}\left(\frac{A}{M}\right) \tilde{g}\left(\frac{A}{M}\right)+\sum_{i=1}^{3} f\left(\frac{1}{Q_{i}^{e_{i}}}\right) g\left(\frac{1}{Q_{i}^{e_{i}}}\right)
$$

Since $(q-1) g(1 / M) \in G_{1}$, we may assume $0 \leq m \leq q-2$. But if $m=0$, then $(q-1) R=0$ implies that $\tilde{f}(A / M)=f\left(1 / Q_{i}^{e_{i}}\right)=0$ by the linear independence (Lemma 3.3), which force $R=0$. Hence we have $1 \leq m \leq q-2$. Now apply the $\operatorname{map} \varphi$ to $R$, then

$$
1=\varphi\left(g\left(\frac{1}{M}\right)\right) \times \prod \varphi\left(\tilde{g}\left(\frac{A}{M}\right)\right)^{\tilde{f}(A / M)} \times \prod \varphi\left(g\left(\frac{1}{Q_{i}^{e_{i}}}\right)\right)^{f\left(1 / Q_{i}^{\varepsilon_{i}}\right)}
$$

Since the first two terms of the right side are units, $f\left(1 / Q_{i}^{e_{i}}\right)=0$ and so we have

$$
R=m g\left(\frac{1}{M}\right)+\sum_{\tilde{g}(A / M) \in G_{1}} \tilde{f}\left(\frac{A}{M}\right) \tilde{g}\left(\frac{A}{M}\right)
$$

with $1 \leq m \leq q-2$. 
For any two nonzero distinct elements $R_{1}, R_{2} \in \operatorname{Tor}\left(\left(\mathrm{A}_{M}^{0}\right)^{+}\right)$, we write

$$
R_{i}=m_{i} g\left(\frac{1}{M}\right)+\sum_{\tilde{g}(A / M) \in G_{i}} \tilde{f}_{i}\left(\frac{A}{M}\right) \tilde{g}\left(\frac{A}{M}\right)
$$

with $1 \leq m_{i} \leq q-2$ as above, then we have $m_{1} \neq m_{2}$. Otherwise,

$$
0=(q-1)\left(R_{1}-R_{2}\right)=\sum_{\tilde{g}(A / M) \in G_{1}}(q-1)\left\{\tilde{f}_{1}\left(\frac{A}{M}\right)-\tilde{f}_{2}\left(\frac{A}{M}\right)\right\} \tilde{g}\left(\frac{A}{M}\right) \in G_{1}
$$

which implies that $R_{1}=R_{2}$.

Since we have $q-2$ nonzero torsion elements in $\left(\mathbf{A}_{M}^{0}\right)^{+}$, we can choose nonzero $R \in \operatorname{Tor}\left(\left(\mathrm{A}_{M}^{0}\right)^{+}\right)$of the desired form. Then we omit the rest of the induction step because it is exactly the same as in the classical case [2].

THEOREM 3.5. $U_{M}=\varphi\left(G_{1}\right) \times \mathbb{F}_{q}^{\times}$, where $\varphi:\left(\mathrm{A}_{M}^{\circ}\right)^{+} \rightarrow V_{M} / \mathbb{F}_{q}^{\times}$is defined as in Section 1.

REMARK. We have shown $\sum_{D}\left|I_{D}^{\prime \prime}\right|=\Phi(M) /(q-1)-1$ in Lemma 3.1. Hence Theorem 3.5 provides a basis of $U_{M}$.

COROLlarY 3.6. Suppose that $M$ and $N$ are monic with $(M, N)=1$. Then

$$
U_{M N}^{G}=U_{M}
$$

where $G=\operatorname{Gal}\left(k\left(\Lambda_{M N}\right) / k\left(\Lambda_{M}\right)\right)$ and $U_{M N}^{G}$ is the subgroup of $U_{M N}$ fixed under the action of $G$.

Now we show that $U_{Q M}^{G}=U_{M}$, where $G=\operatorname{Gal}\left(k\left(\Lambda_{Q M}\right) / k\left(\Lambda_{M}\right)\right)$. When $Q \nmid M$, Corollary 3.6 proves it. So we assume that $Q \mid M$ and let $M=Q_{1}^{e_{1}} \cdots Q_{r}^{e_{r}} Q^{e}$ be the usual factorization of $M$ with $e>0$. Let $S^{*}=S_{r+1}^{*}$ as in Section 2 letting $M=Q_{1}^{e_{1}} \cdots Q_{r}^{e_{r}} Q^{e+1}, Q_{r+1}=Q$, and $K^{*}=\left\{A \in S^{*}: A \equiv 1 \bmod Q^{e}\right\}$. Choose $T^{*} \subset S^{*}$ so that

$$
S^{*}=\bigcup_{A \in T^{*}} A \cdot K^{*}
$$

For each monic divisor $D$ of $M$ such that $(D, M / D)=1,(D, Q)=1$, say $D=$ $\prod_{i=1}^{t} Q_{i_{t}}^{e_{i}}$, we define $\tilde{T}_{D}^{\prime \prime}$ as the group generated by the elements $\tilde{g}\left(A_{i_{1}} \cdots A_{i_{t}} A / D Q^{e+1}\right)$ with $A_{i_{k}} \in S_{i_{k}} \backslash\{1\}(k=1, \ldots, t), A \in S^{*} \backslash T^{*}$ and $\tilde{T}_{M}^{\prime \prime}=\prod_{D} \tilde{T}_{D}^{\prime \prime}$. Then [2, Lemma 5] can be translated as follows: 
LEMMA 3.7. For any $A \in T^{*}$,

$$
\sum_{B \in K^{*}} \tilde{g}\left(\frac{A B}{Q^{e+1}}\right)=\tilde{g}\left(\frac{A}{Q^{e}}\right)+\sum_{B \in K^{*}} \tilde{g}\left(\frac{B}{Q^{e+1}}\right) .
$$

Using Lemma 3.7 we can prove the following theorem.

THEOREM 3.8. $U_{Q M}=\varphi\left(\tilde{T}_{M} \times \tilde{T}_{M}^{\prime} \times \tilde{T}_{M}^{\prime \prime}\right) \times \mathbb{F}_{q}^{\times}$.

PROOF. The proof consists of verifying the following three cases:

(i) $\tilde{g}\left(A_{i_{1}} A_{i_{2}} \cdots A_{i_{1}} A /\left(D Q^{e+1}\right)\right) \in \tilde{T}_{D Q^{*}} \times \tilde{T}_{D Q^{*}}^{\prime} \times \tilde{T}_{D Q^{e}}^{\prime \prime}$ if $A \in S^{*} \backslash T^{*}$.

(ii) $\tilde{g}\left(A_{i_{1}} A_{i_{2}} \cdots A_{i_{i}} A /\left(D Q^{e+1}\right)\right) \in \tilde{T}_{D Q^{*}} \times \tilde{T}_{D Q^{*}}^{\prime} \times \tilde{T}_{D Q^{*}}^{\prime \prime}$ if $A \in T^{*}, A \neq 1$.

(iii) $\tilde{\boldsymbol{g}}\left(A_{i_{1}} A_{i_{2}} \cdots A_{i_{t}} /\left(D Q^{e+1}\right)\right) \in \tilde{T}_{D Q^{*}} \times \tilde{T}_{D Q^{*}}^{\prime} \times \tilde{T}_{D Q^{*}}^{\prime \prime}$

We will omit the proofs because they are very similar to those of [2, Theorem 3] as we did in the proof of Theorem 2.3.

COROLLARY 3.9. Let $Q \mid M$. Then $U_{Q M}^{G}=U_{M}$, where $G=\operatorname{Gal}\left(k\left(\Lambda_{Q M}\right) / k\left(\Lambda_{M}\right)\right)$.

COROLlarY 3.10. Let $M$ and $N$ be monic polynomials such that $M \mid N$. Then the natural map $E_{M} / U_{M} \rightarrow E_{N} / U_{N}$ is an injection.

\section{References}

[1] S. Bae, 'Punctured distributions in function fields', preprint.

[2] R. Gold and J. Kim, 'Bases for cyclotomic units', Compositio Math. 71 (1989), 13-27.

[3] S. Galovich and M. Rosen, 'The class number of cyclotomic function fields', J. Number Theory 13 (1981), 363-375.

[4] - 'Units and class groups in cyclotomic function fields', J. Number Theory 14 (1982), 156-184.

[5] — 'Distributions on rational function fields', Math. Ann. 256 (1981), 549-560.

[6] L. Washington, Introduction to cyclotomic fields (Springer, New York, 1980).

\section{Department of Mathematics}

\section{KAIST}

Yu-song gu, Ku-song dong, 373-1

Taejon, 305-701

Korea

e-mail: shbae@math.kaist.ac.kr

e-mail: hyjung@mathx.kaist.ac.kr 